\title{
Increased Sclerostin, but Not Dickkopf-1 Protein, Is Associated with Elevated Pulse Wave Velocity in Hemodialysis Subjects
}

\author{
Eirini Stavrinou $^{a}$ Pantelis A. Sarafidis $^{a}$ Charalampos Koumaras $^{b}$ \\ Charalampos Loutradis $^{a}$ Panagiotis Giamalis ${ }^{a} \quad$ Konstantinos Tziomalos $^{c}$ \\ Asterios Karagiannis $^{b}$ Aikaterini Papagiannia \\ aDepartment of Nephrology, Hippokration Hospital, Aristotle University of Thessaloniki, \\ Thessaloniki, Greece; ${ }^{b}$ Second Propaedeutic Department of Internal Medicine, Hippokration \\ Hospital, Aristotle University of Thessaloniki, Thessaloniki, Greece; ' ${ }^{\mathrm{C}}$ First Propaedeutic \\ Department of Internal Medicine, AHEPA Hospital, Aristotle University of Thessaloniki, \\ Thessaloniki, Greece
}

\section{Keywords}

Sclerostin · Dickkopf-1 protein · Hemodialysis · Pulse wave velocity · Arterial stiffness

\begin{abstract}
Background: Sclerostin and Dickkopf-1 (Dkk-1) proteins are inhibitors of the canonical Wnt/ $\beta$ catenin bone pathway. Pilot data suggest that sclerostin may be involved in vascular changes in chronic kidney disease (CKD), but data on the effects of Dkk-1 are scarce. This is the first study investigating simultaneously the associations of sclerostin and Dkk-1 with arterial stiffness in hemodialysis patients. Methods: A total of 80 patients on chronic hemodialysis had carotid-femoral pulse wave velocity (PWV), central blood pressure (BP), and wave reflections evaluated with applanation tonometry (Sphygmocor) on a midweek non-dialysis day. Serum levels of sclerostin and Dkk-1 were measured with ELISA. A large set of demographic, comorbid, laboratory, and drug parameters were used in the analyses. Results: Subjects with PWV $>9.5 \mathrm{~m} / \mathrm{s}$ (high arterial stiffness group, $n=40$ ) were older, had higher BMI, higher prevalence of hypertension, diabetes, and coronary heart disease, and higher peripheral systolic BP, central systolic BP, C-reactive protein, and serum sclerostin $(p=0.02)$, but similar Dkk-1, compared to subjects with low PWV. When dichotomizing the population by sclerostin levels, those with high sclerostin had higher PWV than patients with low sclerostin levels (10.63 \pm 2.71 vs. 9.77 $\pm 3.13, p=0.048)$. Increased sclerostin $(>200 \mathrm{pg} / \mathrm{mL}$ ) was significantly associated with increased PWV (>9.5 m/s; HR 2.778, 95\% Cl 1.123-6.868 per pg/mL increase); this association remained significant after stepwise adjustment for Dkk-1, intact parathyroid hormone, and calcium $\times$ phosphate product. In contrast, no association was noted between Dkk-1 and PWV
\end{abstract}




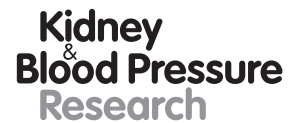

Research

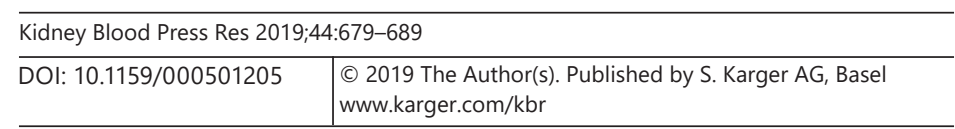

Stavrinou et al.: Sclerostin, Dkk-1, and PWV in Hemodialysis

(HR 1.000, 95\% Cl 0.416-2.403). Conclusion: Serum sclerostin is associated with PWV independently of routine markers of CKD-MBD in hemodialysis patients. In contrast, Dkk-1 has no association with arterial stiffness and is not pathophysiologically involved in relevant vascular changes.

\section{Introduction}

Atherosclerotic and arteriosclerotic vascular changes account to a great extent for the excessive cardiovascular mortality observed in patients with chronic kidney disease (CKD), and particularly those with end-stage renal disease (ESRD) receiving renal replacement therapy $[1,2]$. Pulse wave velocity (PWV), a well-established marker of arteriosclerotic disease, increases in parallel with deterioration of renal function and is associated with traditional cardiovascular risk factors, such as hypertension, diabetes mellitus, and older age, but also with uremia-related disturbances such as arterial calcifications [3, 4]. Arterial calcifications are involved in a complex syndrome of mineral and bone disorders, the CKD-MBD syndrome; they are associated with chronic vascular inflammation and dysregulation of bone turnover biomarkers (such as phosphorus, calcium, bone-specific alkaline phosphatase [bsALP], and several bone proteins), indicating the existence of an association between vascular changes and bone metabolism [5-7].

Sclerostin, the glycoprotein product of the SOST gene, is secreted by osteocytes. It binds to the complex of low-density lipoprotein receptor-related proteins $5 / 6$, which are transmembrane receptors of osteoblasts, and inhibits their Wnt/ $\beta$-catenin signaling pathway, which promotes osteoblast proliferation, maturation, differentiation, and bone formation [8]. Serum sclerostin levels appear to increase with age and as renal function declines [9]. It has been associated with bone biomarkers (intact parathyroid hormone [iPTH], fibroblastgrowth-factor [FGF]-23, bsALP), but also with vascular calcifications and mortality in patients with CKD [10-12]. Dickkopf-1 (Dkk-1) protein also interacts with the complex of receptors LPR5/6 and inhibits the Wnt signaling pathway of the osteoblasts, thereby regulating bone cell differentiation; apart from osteocytes, Dkk-1 is produced by other cell types, such as vascular smooth muscle cells, platelets, and endothelial cells [8, 13-14]. Dkk-1 is likely involved in the interaction between platelets and endothelial cells favoring atherogenesis, whereas clinical evidence supports an association of Dkk-1 with ischemic heart and cerebrovascular disease and coronary artery calcification $[15,16]$. Both inhibitors of the Wnt $/ \beta$ catenin pathway have been implicated in cardiovascular disease by inducing atherogenesis and vascular osteochondrogenesis, besides their role in bone turnover [14].

Studies evaluating the potential relation of Wnt inhibitors with arterial stiffness in ESRD yielded controversial results. In a study including prevalent hemodialysis patients, serum sclerostin was independently associated with PWV only in patients without hyperparathyroidism (iPTH $<300 \mathrm{pg} / \mathrm{mL}$ ) [17]. A positive univariate correlation was demonstrated between sclerostin levels and carotid-femoral PWV in a recent study in hemodialysis patients, but the association lost its significance after adjustment for other confounders [18]. The relation of both Wnt-pathway inhibitors (sclerostin and Dkk-1) with arterial stiffness indices was investigated in a population of postmenopausal women, where a positive correlation between sclerostin but not Dkk-1 with aortic PWV was noted [19], and in a study evaluating the relationship between osteoporosis and cardiovascular disease in 67 ambulatory adults, which again identified sclerostin, but not Dkk-1, as an independent predictor of PWV [20]. 


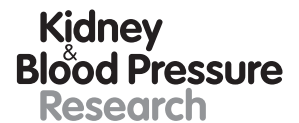

Research

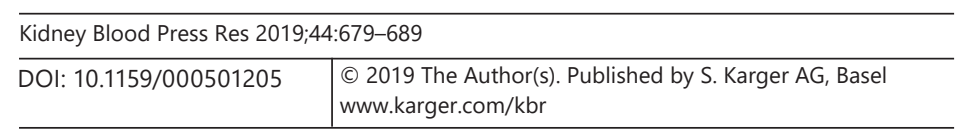

Stavrinou et al.: Sclerostin, Dkk-1, and PWV in Hemodialysis

To the best of our knowledge, no study has examined simultaneously the association of sclerostin and Dkk-1 with PWV in hemodialysis patients. The aim of the present study was to investigate potential correlations of the two Wnt inhibitors with carotid-femoral PWV, in an attempt to explore the complex underlying mechanisms of vascular and bone interactions.

\section{Materials and Methods}

\section{Study Population}

A total of 80 stable ESRD patients receiving standard hemodialysis therapy in the Dialysis Unit of the Department of Nephrology, "Hippokration" University Hospital, Thessaloniki, and in "Evangelismos" Hemodialysis Unit, Imathia, Greece, participated in this study. The study protocol was approved by the Ethics Committee of School of Medicine, Aristotle University of Thessaloniki, and all protocol procedures were conducted in accordance with the Declaration of Helsinki (2008 Amendment). The study was carried out between May 2014 and February 2016 and all subjects provided informed consent prior to study enrollment.

Inclusion criteria consisted of: (i) ESRD under a standard hemodialysis schedule with 3 sessions per week; (ii) the patient being on hemodialysis for at least 3 months prior to study enrollment, and (iii) the patient having provided informed written consent. Patients were excluded from the study in the case of: (i) chronic atrial fibrillation or other cardiac arrhythmia; (ii) presence of old artery-vein anastomoses for earlier arteriovenous fistula formation at the contralateral arm from that currently used for dialysis access interfering with the PWV measurement; (iii) modification of dry weight and antihypertensive treatment during the previous month prior to study enrollment; (iv) stage III-IV congestive heart failure according to the New York Heart Association (NYHA) classification; (v) severe peripheral occlusive arterial disease; (vi) history of acute myocardial infarction or stroke 3 months before the beginning of the study, and (vii) history of parathyroidectomy, active malignancy, infection, or treatment with antibiotics or immunosuppressive agents at the time of the study.

\section{Data Collection and Assessments}

Demographics, anthropometric characteristics, cause of ESRD, comorbidities, concomitant medications, laboratory tests, and dialysis-related parameters were recorded on purpose-built data-collecting sheets. Study measurements were performed during a mid-week non-dialysis day. All subjects were instructed to refrain from smoking, heavy exercise, caffeine, and alcohol consumption for at least $2 \mathrm{~h}$ before the examination. All measurements were performed by a single well-trained physician in the non-fistula arm in a quiet room with a stable air temperature (approximately $22^{\circ} \mathrm{C}$ ) after at least $10 \mathrm{~min}$ of rest in the supine position.

Measurements of peripheral blood pressure (BP) in the branchial artery were performed by a validated mercury sphygmomanometer. Assessment of arterial stiffness and arterial wave parameters were performed with the use of a Sphygmocor device. Applanation tonometry with a high-fidelity pencil-type probe (SPT-301, Millar Instruments, Houston, TX, USA) on the radial artery connected to a computer with Sphygmocor software (AtCor, Sydney, Australia) was performed to measure aortic pressures and arterial wave parameters $[21,22]$. The Sphygmocor software reconstructs the aortic pulse waveform by using a validated generalized transfer function. We introduced brachial BP for the calibration of the aortic pulse waveform, measured immediately before the Sphygmocor recording, with a mercury sphygmomanometer in the non-fistula arm, according to current guidelines [23]. Augmentation pressure (AP) was defined as the difference of aortic pressures between the second and first systolic peaks. The augmentation index (AIx) was calcu- 


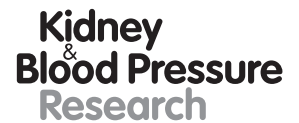

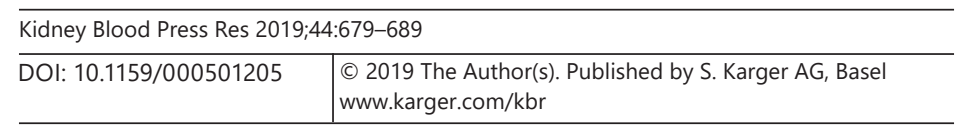

Stavrinou et al.: Sclerostin, Dkk-1, and PWV in Hemodialysis

lated by dividing AP by aortic pulse pressure (PP) and was expressed as a percentage, whereas augmentation index adjusted for heart rate 75 beats/min (AIx 75) was estimated by the Sphygmocor software by adjusting AIx at an inverse rate of $4.8 \%$ for every 10 beats/ min elevation in heart rate.

Aortic PWV was measured by performing applanation tonometry and recording pulse waveforms at the carotid and femoral arteries with the above-described pencil-type tonometer. Pulse waveforms were referenced to a concurrently recorded ECG, and pulse wave transit time between the subsequent recording sites was calculated from the Sphygmocor software, according to the foot-to-foot time difference between carotid/femoral waveforms. Body surface distances from the suprasternal notch to the carotid recording site (distance A), and from the suprasternal notch to the femoral recording site (distance B) were measured, and pulse wave travel distance was calculated by subtracting the distance A from distance B. Aortic PWV was calculated as the pulse wave travel distance in meters divided by the pulse wave transit time in seconds [21,22]. We recorded PWV over 10 consecutive heartbeats to cover a whole respiratory cycle. The device's quality control criteria for optimal pressure waveform recording were applied; the first valid recording was used in the subsequent analysis.

\section{Laboratory Analyses}

Blood samples for each participant were drawn from a peripheral vein under fasting conditions in the morning of a midweek routine dialysis session. Serum samples were separated from clotted blood by immediate centrifugation $(1,500 \mathrm{~g}$ for $10 \mathrm{~min})$, aliquoted and stored at $-70{ }^{\circ} \mathrm{C}$ until assay. Serum levels of sclerostin and Dkk-1 were measured by an enzyme-linked immunosorbent assay (ELISA) using commercially available standard kits (human SOST Quantakine, human Dkk-1 Quantakine, Bio-techne, R\&D Systems; detection limit 1.74 and $4.2 \mathrm{pg} / \mathrm{mL}$, respectively). Serum albumin, total cholesterol, triglycerides, HDL cholesterol, LDL cholesterol, calcium, phosphorus, and ALP were determined by routine techniques using an automated analyzer (Olympus AU560, Hamburg, Germany). Levels of albumin were time averaged for the last 6 months before inclusion in the study. iPTH levels were measured by radioimmunoassay. Serum C-reactive protein levels were measured by nephelometry.

\section{Statistical Analysis}

Statistical analysis was performed using the Statistical Package for Social Sciences version 23.0 (SPSS Inc., Chicago, IL, USA). Values of $p<0.05$ (two-tailed) were considered statistically significant for all comparisons. The Shapiro-Wilk or the Kolmogorov-Smirnov test were applied to examine the normality of the distribution for continuous variables. Quantitative variables are presented as the mean \pm standard deviation, or median and range, and qualitative variables as frequencies and percentages. Comparisons of continuous parameters between the groups of interest were performed with paired Student $t$ tests for independent variables or the Mann-Whitney test, where appropriate, depending on the normality of the distribution. The $\chi^{2}$ test or the Fisher exact test were used for between-group comparisons for categorical variables. To evaluate the possible associations between parameters under study and sclerostin or Dkk-1 levels, Spearman's rho or Pearson's $r$ correlation coefficients were calculated. Finally, to examine to possible impact of various factors on the association between sclerostin or Dkk-1 and high PWV values, stepwise logistic regression modeled analysis (enter method) was performed. 
Table 1. Clinical characteristics and laboratory parameters of 80 hemodialysis patients with high or low PWV

\begin{tabular}{|c|c|c|c|c|}
\hline & All patients $(n=80)$ & $P W V \leq 9.5$ & $\mathrm{PWV}>9.5 \mathrm{~m} / \mathrm{s}$ & $p$ \\
\hline$n$ & $80(100)$ & $40(50.0)$ & $40(50.0)$ & - \\
\hline Age, years & $60.9 \pm 13.7$ & $53.8 \pm 13.5$ & $68.1 \pm 9.6$ & $<0.001$ \\
\hline Female & $35(43.8)$ & $18(45.0)$ & $17(42.5)$ & 0.822 \\
\hline Weight, kg & $71.7 \pm 14.2$ & $68.7 \pm 13.1$ & $74.8 \pm 14.9$ & 0.054 \\
\hline Height, $\mathrm{cm}$ & $167.6 \pm 9.3$ & $168.1 \pm 9.0$ & $167.1 \pm 9.7$ & 0.635 \\
\hline BMI & $25.3 \pm 4.6$ & $24.1 \pm 4.3$ & $26.5 \pm 4.6$ & 0.018 \\
\hline Dialysis vintage, months & $36.5(3-213)$ & $41(4-180)$ & $26(3-213)$ & 0.214 \\
\hline $\mathrm{Kt} / \mathrm{V}$ & $1.5 \pm 0.3$ & $1.5 \pm 0.2$ & $1.5 \pm 0.4$ & 0.713 \\
\hline Diabetes mellitus & $17(21.3)$ & $2(5.0)$ & $15(37.5)$ & $<0.001$ \\
\hline Hypertension & $57(71.3)$ & $23(57.5)$ & $34(85.0)$ & 0.007 \\
\hline Coronary heart disease & $17(21.3$ & $4(10.0)$ & $13(32.5)$ & 0.014 \\
\hline Peripheral vascular disease & $6(7.5)$ & $2(5.0)$ & $4(10.0)$ & 0.396 \\
\hline Stroke & $1(1.3)$ & $0(0.0)$ & $1(2.5)$ & 0.314 \\
\hline Smoking & $16(20.0)$ & $8(20.0)$ & $8(20.0)$ & 1.000 \\
\hline Hemoglobin, g/dL & $11.2 \pm 0.9$ & $11.4 \pm 0.8$ & $11.0 \pm 0.9$ & 0.025 \\
\hline Serum urea, $\mathrm{mg} / \mathrm{dL}$ & $125.4 \pm 25.3$ & $128.5 \pm 19.6$ & $122.4 \pm 30.0$ & 0.283 \\
\hline Serum creatinine, $\mathrm{mg} / \mathrm{dL}$ & $7.6 \pm 2.1$ & $8.3 \pm 2.1$ & $6.9 \pm 1.9$ & 0.003 \\
\hline Serum calcium, mg/dL & $8.9 \pm 0.6$ & $9.0 \pm 0.6$ & $8.8 \pm 0.5$ & 0.118 \\
\hline Serum phosphate, mg/dL & $4.8 \pm 1.1$ & $4.6 \pm 1.0$ & $5.0 \pm 1.3$ & 0.173 \\
\hline Serum alkaline phosphatase, $U / L$ & $173.2 \pm 95.5$ & $160.4 \pm 94.2$ & $186.1 \pm 96.3$ & 0.231 \\
\hline Albumin, g/dL & $4.1 \pm 0.3$ & $4.1 \pm 0.3$ & $4.0 \pm 0.4$ & 0.069 \\
\hline CRP, mg/L & $3.49(3.00-142.00)$ & $3.38(3.00-32.70)$ & $4.00(3.00-142.00)$ & 0.025 \\
\hline iPTH, ng/L & $323.00(70.00-1633.00)$ & $328.00(88.00-1200.00)$ & $315.00(70.00-1,633.00)$ & 0.603 \\
\hline Sclerostin, $\mathrm{pg} / \mathrm{mL}$ & $201.00(45.16-828.50)$ & $183.50(46.83-710.16)$ & $251.83(45.16-828.50)$ & 0.020 \\
\hline Dkk-1,pg/mL & $339.25(9.25-1,379.00)$ & $339.25(143.00-1,379.00)$ & $353.00(9.25-735.50)$ & 0.644 \\
\hline Paricalcitol & $42(52.5)$ & $19(47.5)$ & $23(57.5)$ & 0.370 \\
\hline Active vitamin D & $5(6.3)$ & $2(5.0)$ & $3(7.5)$ & 0.644 \\
\hline Calcimimetics & $18(22.5)$ & $9(22.5)$ & $9(22.5)$ & 1.000 \\
\hline Sevelamer & $49(61.3)$ & $27(67.5)$ & $22(55.0)$ & 0.251 \\
\hline Lanthanum & $28(35.0)$ & $16(40.0)$ & $12(30.0)$ & 0.348 \\
\hline Oral calcium supplement & $12(15.0)$ & $8(20.0)$ & $4(10.0)$ & 0.210 \\
\hline ARBs & $5(6.3)$ & $1(2.5)$ & $4(10.0)$ & 0.359 \\
\hline ACEIs & $7(8.8)$ & $5(12.5)$ & $2(5.0)$ & 0.432 \\
\hline CCBs & $27(33.8)$ & $9(22.5)$ & $18(45.0)$ & 0.033 \\
\hline$\beta$-Blockers & $45(56.3)$ & $18(45.0)$ & $27(67.5)$ & 0.043 \\
\hline Statins & $28(35.0)$ & $11(27.5)$ & $17(42.5)$ & 0.160 \\
\hline ESAs & 69 (86.3) & $33(82.5)$ & $36(90.0)$ & 0.330 \\
\hline SBP, mm Hg & $136.3 \pm 19.1$ & $129.3 \pm 17.9$ & $143.3 \pm 17.9$ & 0.001 \\
\hline DBP, mm Hg & $79.5 \pm 10.0$ & $78.3 \pm 9.8$ & $80.7 \pm 10.1$ & 0.269 \\
\hline $\mathrm{PP}, \mathrm{mm} \mathrm{Hg}$ & $56.8 \pm 16.9$ & $51.1 \pm 13.8$ & $62.5 \pm 17.8$ & 0.002 \\
\hline cSBP, mm Hg & $127.4 \pm 18.4$ & $120.4 \pm 17.1$ & $134.5 \pm 17.2$ & $<0.001$ \\
\hline cDBP, mm Hg & $80.0 \pm 10.7$ & $78.6 \pm 11.3$ & $81.4 \pm 10.0$ & 0.244 \\
\hline $\mathrm{cPP}, \mathrm{mm} \mathrm{Hg}$ & $47.5 \pm 16.2$ & $41.8 \pm 13.9$ & $53.1 \pm 16.4$ & 0.001 \\
\hline AIx, \% & $31.8 \pm 10.3$ & $30.6 \pm 12.0$ & $33.0 \pm 8.3$ & 0.315 \\
\hline Augmentation pressure, $\mathrm{mm} \mathrm{Hg}$ & $15.7 \pm 8.2$ & $13.2 \pm 7.1$ & $18.2 \pm 8.6$ & 0.006 \\
\hline AIx $75, \%$ & $30.2 \pm 9.6$ & $29.4 \pm 10.9$ & $31.1 \pm 8.1$ & 0.445 \\
\hline Heart rate, beats/min & $72.1 \pm 9.0$ & $73.8 \pm 9.5$ & $70.4 \pm 8.2$ & 0.091 \\
\hline
\end{tabular}

Values are expressed as the mean \pm SD or $n(\%)$. Bold $p$ values are significant. CRP, C-reactive protein; Dkk-1, Dickkopf-1 protein; iPTH, intact parathyroid hormone; Dkk-1, Dickkopf-related protein; ARBs, angiotensin II receptor blockers; ACEIs, angiotensin converting enzyme inhibitors; CCBs, calcium channel blockers; ESAs, erythropoietin-stimulating agents; SBP, systolic blood pressure; DBP, diastolic blood pressure; PP, pulse pressure; cSBP, central systolic blood pressure; cDBP, central diastolic blood pressure; cPP, central pulse pressure; AIx, augmentation index; AIx 75, augmentation index adjusted for heart rate 75 beats/min.

\section{Results}

\section{Characteristics of Study Participants}

Table 1 depicts the demographic characteristics, routine laboratory data, dialysis-related parameters, and drug treatment of the study participants. A total of 45 men and 35 women with a mean age $60.9 \pm 13$ years, and on standard hemodialysis therapy for a median duration of 36.5 (3-213) months, were included. The primary causes of ESRD were glomerulonephritis $(22,5 \%)$, diabetes mellitus (21\%), tubulointerstitial nephritis (11\%), polycystic kidney disease (14\%), vascular disease $(8,7 \%)$, and undetermined $(22,5 \%)$. After dichotomizing our study population 
Table 2. Univariate correlation analysis of sclerostin and Dkk-1 with demographic characteristics and bone and vascular markers

\begin{tabular}{|c|c|c|c|c|}
\hline & \multicolumn{2}{|c|}{ Sclerostin } & \multicolumn{2}{|l|}{ Dkk-1 } \\
\hline & $r$ & $p$ & $r$ & $p$ \\
\hline Age (years) & 0.439 & $<0.001$ & -0.223 & 0.047 \\
\hline BMI & 0.270 & 0.015 & 0.027 & 0.811 \\
\hline Dialysis vintage (months) & -0.122 & 0.283 & -0.146 & 0.196 \\
\hline Hemoglobin $(\mathrm{g} / \mathrm{dL})$ & -0.103 & 0.365 & -0.024 & 0.834 \\
\hline Serum urea $(\mathrm{mg} / \mathrm{dL})$ & 0.016 & 0.885 & 0.070 & 0.536 \\
\hline Serum creatinine $(\mathrm{mg} / \mathrm{dL})$ & -0.012 & 0.918 & 0.157 & 0.163 \\
\hline Serum calcium (mg/dL) & -0.162 & 0.152 & 0.153 & 0.175 \\
\hline Serum phosphate (mg/dL) & 0.087 & 0.442 & 0.123 & 0.277 \\
\hline Serum alkaline phosphatase (U/L) & -0.094 & 0.405 & -0.154 & 0.174 \\
\hline Intact parathormone $(\mathrm{ng} / \mathrm{L})$ & -0.314 & 0.005 & 0.046 & 0.684 \\
\hline Albumin $(g / d L)$ & 0.049 & 0.664 & 0.153 & 0.175 \\
\hline Cholesterol (mg/dL) & -0.027 & 0.812 & -0.056 & 0.619 \\
\hline LDL (mg/dL) & 0.076 & 0.503 & -0.058 & 0.606 \\
\hline Triglycerides (mg/dL) & 0.147 & 0.194 & 0.013 & 0.909 \\
\hline $\mathrm{CRP}(\mathrm{mg} / \mathrm{L})$ & 0.134 & 0.236 & -0.053 & 0.642 \\
\hline SBP (mm Hg) & 0.083 & 0.466 & 0.040 & 0.725 \\
\hline DBP (mm Hg) & -0.043 & 0.708 & 0.190 & 0.091 \\
\hline $\mathrm{PP}(\mathrm{mm} \mathrm{Hg})$ & 0.116 & 0.304 & -0.058 & 0.607 \\
\hline cSBP (mm Hg) & 0.037 & 0.742 & 0.051 & 0.652 \\
\hline cDBP (mm Hg) & -0.054 & 0.634 & 0.137 & 0.226 \\
\hline cPP (mm Hg) & 0.082 & 0.468 & -0.011 & 0.924 \\
\hline $\mathrm{HR}(\mathrm{bpm})$ & -0.133 & 0.241 & -0.084 & 0.458 \\
\hline $\operatorname{AIx}(\%)$ & -0.105 & 0.352 & 0.036 & 0.750 \\
\hline Augmentation pressure (mm Hg) & -0.034 & 0.765 & 0.069 & 0.545 \\
\hline AIx $75(\%)$ & -0.135 & 0.233 & -0.024 & 0.829 \\
\hline $\mathrm{PWV}(\mathrm{m} / \mathrm{s})$ & 0.207 & 0.065 & -0.045 & 0.694 \\
\hline Dkk-1 (pg/mL) & -0.146 & 0.197 & & \\
\hline
\end{tabular}

Bold $p$ values are significant. LDL, low-density lipoprotein; CRP, C-reactive protein; SBP, systolic blood pressure; DPB, diastolic blood pressure; PP, pulse pressure; cSBP, central systolic blood pressure; cDBP, central diastolic blood pressure; cPP, central pulse pressure; HR, heart rate; AIx, aortic augmentation index; AIx 75, augmentation index adjusted for heart rate 75 beats/min; PWV, pulse wave velocity, Dkk-1, Dickkopf-1 protein.

based on the median PWV $(9.5 \mathrm{~m} / \mathrm{s})$, there were no differences between the two groups for sex, dialysis vintage, and routine biochemical parameters of bone metabolism. However, patients in the high PWV group were older (53.8 \pm 13.5 vs. $68.1 \pm 9.6, p<0.001)$, had higher BMI $(26.5 \pm 4.6$ vs. $24.1 \pm 4.3, p=0.018$ ), and more frequently had diabetes ( 37.5 vs. $5 \%, p<0.001$ ), hypertension ( 85 vs. $57.5 \%, p=0.007$ ), and coronary heart disease (32.5 vs. $10 \%, p=0.014$ ). Compared with the low PWV group, patients in the high PWV also had significantly higher peripheral and central systolic BP, peripheral and central PP, AP, and C-reactive protein. Of importance, serum sclerostin levels were higher in patients in the high PWV group (251.83 vs. $183.50, p=0.020$ ), whereas Dkk-1 protein levels were similar between the two groups (353.00 vs. 339.25, $p=$ $0.644)$.

Associations of Serum Sclerostin and Serum Dkk-1 Protein with Bone and Vascular Markers

To identify possible associations of the two Wnt pathway inhibitors with basic demographic characteristics, as well as bone and vascular markers, exploratory univariate correlation analyses were first performed. As shown in Table 2, sclerostin was associated posi- 
Table 3. Modeled logistic regression analysis using PWV as the dependent variable (categorical variable for the median, $>9.5$ cut-off point) and sclerostin (categorical variable for the median; $>200 \mathrm{pg} / \mathrm{mL}$ cut-off point) or Dkk-1 (categorical variable for the median; $>340 \mathrm{pg} / \mathrm{mL}$ cut-off point) as the independent variable

\begin{tabular}{|c|c|c|c|c|c|c|}
\hline & \multicolumn{3}{|c|}{ a Sclerostin } & \multicolumn{3}{|c|}{ b Dkk-1 } \\
\hline & OR & $95 \%$ CI & $p$ & OR & $95 \%$ CI & $p$ \\
\hline Model 1 & 2.778 & $1.123-6.868$ & 0.027 & 1.000 & $0.416-2.403$ & 1.000 \\
\hline Model 2 & 2.677 & $1.072-6.684$ & 0.035 & 1.109 & $0.450-2.734$ & 0.822 \\
\hline Model 3 & 2.769 & $1.090-7.037$ & 0.032 & 1.106 & $0.448-2.730$ & 0.827 \\
\hline Model 4 & 2.813 & $1.098-7.209$ & 0.031 & 1.023 & $0.403-2.599$ & 0.962 \\
\hline Model 5 & 1.333 & $0.348-5.097$ & 0.675 & 1.053 & $0.284-3.901$ & 0.939 \\
\hline
\end{tabular}

a Model 1, unadjusted; Model 2, adjusted for Dkk-1; Model 3, adjusted for Dkk-1 and iPTH; Model 4, adjusted for Dkk-1,iPTH, and calcium phosphate product; Model 5, adjusted for Dkk-1, iPTH, calcium phosphate product, age, gender, BMI, hemodialysis vintage, diabetes, hypertension, and cardiovascular disease (defined as a history of coronary heart disease, stroke, and/or peripheral vascular disease).

b Model 1, unadjusted; Model 2, adjusted for sclerostin; Model 3, adjusted for sclerostin and iPTH; Model 4, adjusted for sclerostin, iPTH, and calcium phosphate product; Model 5, adjusted for sclerostin, iPTH, calcium phosphate product, age, gender, BMI, hemodialysis vintage, diabetes, hypertension, and cardiovascular disease (defined as a history of coronary heart disease, stroke, and/or peripheral vascular disease).

Fig. 1. $P W V(m / s)$ according to levels of sclerostin and Dkk-1.

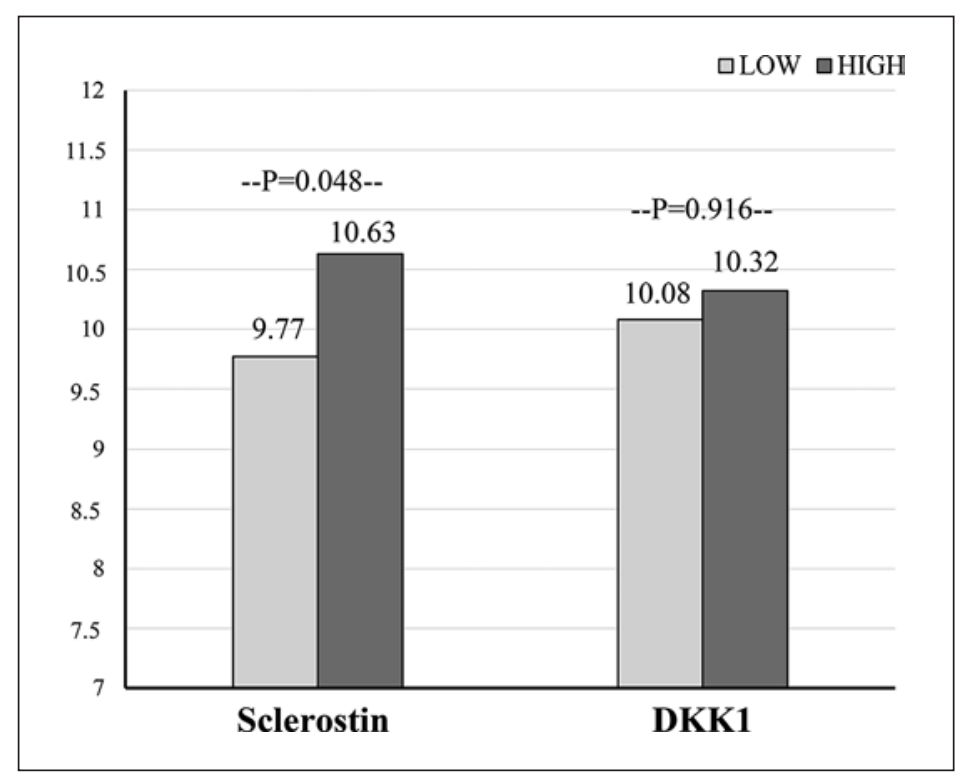

tively with age ( $r=0.439, p<0.001)$, BMI $(r=0.270, p=0.015)$, and negatively with iPTH $(r=-0.314, p=0.005)$. In addition, the univariate association of sclerostin with PWV was marginally significant $(r=0.207, p=0.065)$. In contrast to sclerostin, Dkk-1 only showed a weak inverse association with age $(r=-0.223, p=0.047)$ and no further significant correlations with any marker of vascular disease or bone metabolism.

\section{PWV according to Levels of Serum Sclerostin and Dkk-1 Protein}

Figure 1 presents $\mathrm{PWV}$ in two groups generated by dichotomizing our study population according to serum sclerostin levels, i.e., patients with high (mean $369.41 \pm 174.06 \mathrm{pg} / \mathrm{mL}$ ) 


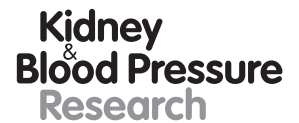

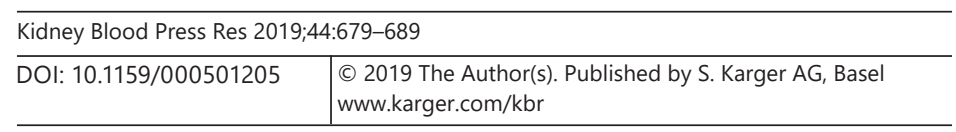

Stavrinou et al.: Sclerostin, Dkk-1, and PWV in Hemodialysis

and patients with low (mean $135.83 \pm 45.44 \mathrm{pg} / \mathrm{mL}$ ) serum sclerostin. As shown, patients with high serum sclerostin had significantly higher PWV levels than patients with low serum sclerostin $(10.63 \pm 2.71$ vs. $9.77 \pm 3.13, p=0.048)$. In contrast to the above, when dividing the population into patients with high (mean $512.64 \pm 180.24 \mathrm{pg} / \mathrm{mL}$ ) and those with low $(224.79$ $\pm 87.30 \mathrm{pg} / \mathrm{mL}$ ) Dkk-1 levels, no difference between the two groups in PWV was noted (10.08 \pm 2.75 vs. $10.32 \pm 3.16, p=0.916$ ) , as depicted in Figure 1 .

\section{Determinants of the Associations of Increased Sclerostin or Dkk-1 with High PWV}

In Table 3, we present the stepwise logistic regression modeled analysis for the association between high sclerostin or Dkk-1 and high PWV levels (>9.5 m/s). In this analysis, the main independent parameter of interest was examined as a dichotomous variable using the median value as the cut-off point. Increased sclerostin levels ( $>200 \mathrm{pg} / \mathrm{mL}$ ) were significantly associated with increased PWV levels (i.e., HR 2.778, 95\% CI 1.123-6.868 per pg/mL increase; Model 1, Table 3a). This association remained significant and with similar HRs after stepwise adjustment for Dkk-1 (Model 2), Dkk-1 and iPTH (Model 3), and Dkk-1, iPTH, and calcium $\times$ phosphate product (Model 4: HR 2.813, 95\% CI 1.098-7.209 per pg/mL increase). Naturally, this association was rendered insignificant only when several other parameters theoretically associated with arterial stiffness and (age, gender, BMI, hemodialysis vintage, diabetes, hypertension, and cardiovascular disease) were included in the model. In contrast to the above, high Dkk-1 was not associated with high PWV levels even in the univariate model (i.e., HR 1.000, 95\% CI 0.416-2.403 per pg/mL increase; Table 3b). The association between Dkk-1 and PWV remained unchanged even after adjustment for sclerostin and all routine laboratory markers of CKD-MBD (Models 2-4), or after adjusting for all additional cardiovascular factors (Model 5).

\section{Discussion}

This is the first study investigating simultaneously the associations of sclerostin and Dkk-1, two inhibitors of the canonical Wnt/ $\beta$-catenin pathway, with arterial stiffness in hemodialysis patients. Patients with high serum sclerostin had significantly higher PWV levels than patients with low serum sclerostin, whereas patients with high and low Dkk-1 levels had no difference in PWV values. Increased sclerostin levels $(>200 \mathrm{pg} / \mathrm{mL})$ were significantly associated with increased PWV ( $>9.5 \mathrm{~m} / \mathrm{s})$; this association remained significant after stepwise adjustment for Dkk-1, iPTH, and calcium $\times$ phosphate product. In contrast, no association was noted between Dkk-1 and PWV. In correlation analyses, serum sclerostin was positively associated with age and BMI, and negatively with iPTH, whereas Dkk-1 protein showed only a weak inverse relation with age.

Current evidence suggests that regulatory proteins of bone metabolism, such as Wnt/ $\beta$ catenin pathway inhibitors, sclerostin, and Dkk-1, participate in the pathogenesis of extraskeletal calcification with a potential impact on arteriosclerotic vascular damage and adverse outcomes [21, 24, 25]. Sclerostin, an osteocyte-derived inhibitor of the Wnt signaling pathway, is a potent suppressor of bone formation, by inhibiting osteoblast differentiation and inducing their apoptosis. In calcified vessels, sclerostin expression is upregulated by osteoblast-like cells as a potential defensive response against the progression of calcification, resulting in increased levels of sclerostin in circulation. Available data suggest that circulating sclerostin is higher in CKD patients and increases progressively as renal function declines [10, 11]. The present study showed a positive association of serum sclerostin with age and an inverse correlation with iPTH, which are in accordance with findings from the relevant literature, as sclerostin production is suppressed by iPTH [26]. In previous studies, significant 


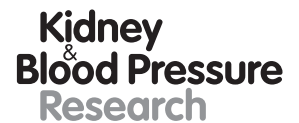

Kidney
Blood Pressure
Research \begin{tabular}{l|l}
\hline Kidney Blood Press Res 2019;44:679-689 \\
\hline DOI: 10.1159/000501205 & $\begin{array}{l}\text { @ 2019 The Author(s). Published by S. Karger AG, Basel } \\
\text { www.karger.com/kbr }\end{array}$ \\
\hline
\end{tabular}

Stavrinou et al.: Sclerostin, Dkk-1, and PWV in Hemodialysis

correlations were demonstrated between serum sclerostin and other biomarkers of bone metabolism in CKD, such as serum phosphorus, bsALP, and FGF-23 [27, 28]; however, these findings are not consistent in all studies, possibly due to discrepancies in the available sclerostin assays or other limitations [29].

Data from studies evaluating the potential relation of sclerostin with arterial stiffness indices in hemodialysis are limited and yield controversial results. In 154 prevalent hemodialysis patients, serum sclerostin showed a weak univariate correlation with carotidfemoral PWV $(r=0.162, p=0.044)$; this association was stronger in patients with iPTH $<300$ $\mathrm{pg} / \mathrm{mL}(r=0.270, p=0.009)$. Sclerostin levels were higher in patients with high than those with low arterial stiffness, but even in the latter group sclerostin was an independent predictor of PWV [17]. The authors suggested that low bone turnover, caused by decreased iPTH levels, promotes extraosseous vascular calcifications, leading to increased arterial stiffness. Similarly, a positive correlation was demonstrated between sclerostin levels and carotid-femoral PWV in 97 hemodialysis patients $(r=0.286, p=0.019)$. After stratifying patients by levels of PWV ( $>9 \mathrm{~m} / \mathrm{s})$, sclerostin was higher in patients with high arterial stiffness (368.85 vs. $286.78 \mathrm{pg} / \mathrm{mL}$ ) [18]. Our study results are in direct agreement with the above, showing a marginally significant association of the same magnitude between sclerostin and PWV $(r=0.207, p=0.065)$ and higher PWV in patients with higher sclerostin. We went a step further to perform a careful stepwise regression analysis to show that the association of high sclerostin with high PWV is strong and independent from Dkk-1, iPTH, and calcium $\times$ phosphate product.

No study has evaluated the associations of both sclerostin and Dkk-1 in the same hemodialysis population. A study of the association between bone loss and vascular changes in 146 postmenopausal women observed different sclerostin (23.8 vs. $29.7 \mathrm{pmol} / \mathrm{L}, p=0.003$ ) but not Dkk-1 levels in the two groups of PWV (lower and higher than $9 \mathrm{~m} / \mathrm{s}$ ) [19], as in our study. A study in 67 subjects (mean age $67.4 \pm 9.0$ years) investigating pathogenetic factors for atherosclerosis and osteoporosis showed sclerostin to be associated with PWV in a multilinear regression model ( $\beta=-0.1472, p=0.0023)$, whereas Dkk- 1 was not [20]. In a crosssectional study of 68 renal transplant recipients, sclerostin but not Dkk-1 was also significantly associated with arterial stiffness [30]. Importantly, in most of the above studies, the specific associations of Dkk-1 with PWV are not reported. The only study showing an association of Dkk-1 with arterial stiffness included 77 patients with CKD stages $3 \mathrm{~b}$ and 4 . Therein, Dkk-1 levels were inversely related to the arterial stiffness index $(r=-0.287, p=0.013)$, whereas sclerostin levels were not; this study was limited by the fact that arterial stiffness was assessed from contour analysis of digital volume pulse and not carotid-femoral PWV [31]. Overall, our findings are in line with the majority of the above studies, showing that Dkk-1 levels had no association with PWV.

Current data suggest that Dkk-1 is involved in the development of focal intimal calcification in atherosclerotic disease, rather than in the more generalized process of medial calcification, which is the major determinant of PWV [13]. In contrast, in experimental studies sclerostin expression was increased at medial vascular calcification sites [32], while in ours and the aforementioned studies its serum levels were associated with PWV. Further explaining the discrepancy in the association of the two Wnt inhibitors with arterial stiffening, data have showed that unlike sclerostin, Dkk-1 is expressed not only by osteogenic vascular cells, but also by endothelial cells, inflammatory cells, and platelets, also within the atherosclerotic plaque. The possibility that the two inhibitors are involved in different processes of vascular pathophysiology is also supported by studies indicating a positive association between serum sclerostin and arterial medial disease (i.e., in type 2 diabetes) [33], and studies suggesting an inverse relationship between Dkk-1 and calcified plaque score [16]. Overall, future studies are needed to investigate the exact role of Dkk-1 in extraosseous ossification. 


\section{Kidney \\ Blood Pressure Research}

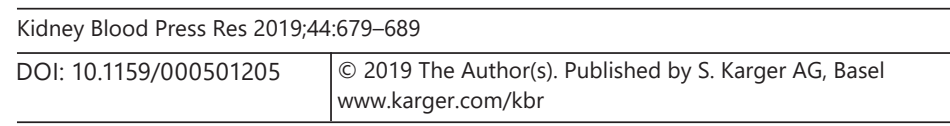

tavrinou et al.: Sclerostin, Dkk-1, and PWV in Hemodialysis

Although this study followed a careful design and analyses, and used the gold standard method for PWV assessment, there are some limitations that need to be acknowledged. First, this is a cross-sectional study that does not allow the identification of causal relationships between serum sclerostin and aortic stiffness. Second, the number of subjects was relatively small, but this did not affect our main findings, as the basic observations are rather clear in terms of statistical significance. We did not include other important bone biomarkers in our models, such as 1,25(OH)2D levels, which were evaluated in some studies and showed to be correlated with sclerostin $[27,28]$. A previous study suggested a seasonal variation in sclerostin levels of about $20 \%$ throughout the year; baseline evaluation of our patients was not performed within a small time frame but was reasonably distributed (between 19 and $34 \%$ ) in the different seasons. Finally, we used the traditional method to estimate carotidfemoral distance (subtracting the distance from the carotid location of measurement to the sternal notch from the distance between the sternal notch and the femoral site of measurement) [21], which may overestimate the true carotid-femoral distance.

In conclusion, the present study showed that serum sclerostin is associated with PWV independently of routine markers of CKD-MBD, such as iPTH and calcium $\times$ phosphate product, in hemodialysis patients. As in other studies, sclerostin correlated positively with age and negatively with serum iPTH. In contrast, Dkk-1 had no association with arterial stiffness in any of the analyses performed. Overall, Dkk-1 levels showed only a weak inverse association with age. Thus, Dkk-1 is rather a bystander that is not pathophysiologically involved in arteriosclerotic (medial) vascular changes of CKD-MBD and could have a role in intimal disease. Further studies are required to fully elucidate the exact mechanisms of the relationship between serum sclerostin and arteriosclerosis in patients with CKD.

\section{Statement of Ethics}

The study protocol was approved by the Ethics Committee of School of Medicine, Aristotle University of Thessaloniki, and all protocol procedures were conducted in accordance with the Declaration of Helsinki (2008 Amendment).

\section{Disclosure Statement}

All authors disclose that they do not have any financial or other relationships which might lead to a conflict of interest. This paper was not supported by any source and represents the original work of the authors.

\section{References}

1 Tonelli M, Wiebe N, Culleton B, House A, Rabbat C, Fok M, et al. Chronic kidney disease and mortality risk: a systematic review. J Am Soc Nephrol. 2006 Jul;17(7):2034-47.

2 Go AS, Chertow GM, Fan D, McCulloch CE, Hsu CY. Chronic kidney disease and the risks of death, cardiovascular events, and hospitalization. N Engl J Med. 2004 Sep;351(13):1296-305.

3 Ford ML, Tomlinson LA, Chapman TP, Rajkumar C, Holt SG. Aortic stiffness is independently associated with rate of renal function decline in chronic kidney disease stages 3 and 4. Hypertension. 2010 May;55(5):1110-5.

4 Verbeke F, Van Biesen W, Honkanen E, Wikström B, Jensen PB, Krzesinski JM, et al.; CORD Study Investigators. Prognostic value of aortic stiffness and calcification for cardiovascular events and mortality in dialysis patients: outcome of the calcification outcome in renal disease (CORD) study. Clin J Am Soc Nephrol. 2011 Jan;6(1): 153-9.

5 Giachelli CM. The emerging role of phosphate in vascular calcification. Kidney Int. 2009 May;75(9):890-7.

6 Cannata-Andía JB, Rodríguez-García M, Carrillo-López N, Naves-Díaz M, Díaz-López B. Vascular calcifications: pathogenesis, management, and impact on clinical outcomes. J Am Soc Nephrol. 2006 Dec;17(12 Suppl 3):S26773. 


\begin{tabular}{l|l}
\hline Kidney Blood Press Res 2019;44:679-689 \\
\hline DOI: 10.1159/000501205 & $\begin{array}{l}\text { (c) 2019 The Author(s). Published by S. Karger AG, Basel } \\
\text { www.karger.com/kbr }\end{array}$ \\
\hline
\end{tabular}

Stavrinou et al.: Sclerostin, Dkk-1, and PWV in Hemodialysis

7 Naves M, Rodríguez-García M, Díaz-López JB, Gómez-Alonso C, Cannata-Andía JB. Progression of vascular calcifications is associated with greater bone loss and increased bone fractures. Osteoporos Int. 2008 Aug;19(8):1161-6.

8 Monroe DG, McGee-Lawrence ME, Oursler MJ, Westendorf JJ. Update on Wnt signaling in bone cell biology and bone disease. Gene. 2012 Jan;492(1):1-18.

9 Pelletier S, Dubourg L, Carlier MC, Hadj-Aissa A, Fouque D. The relation between renal function and serum sclerostin in adult patients with CKD. Clin J Am Soc Nephrol. 2013 May;8(5):819-23.

10 Moysés RM, Schiavi SC. Sclerostin, Osteocytes, and Chronic Kidney Disease - Mineral Bone Disorder. Semin Dial. 2015 Nov-Dec;28(6):578-86.

11 Drechsler C, Evenepoel P, Vervloet MG, Wanner C, Ketteler M, Marx N, et al.; NECOSAD Study Group. High levels of circulating sclerostin are associated with better cardiovascular survival in incident dialysis patients: results from the NECOSAD study. Nephrol Dial Transplant. 2015 Feb;30(2):288-93.

12 Kanbay M, Siriopol D, Saglam M, Kurt YG, Gok M, Cetinkaya H, et al. Serum sclerostin and adverse outcomes in nondialyzed chronic kidney disease patients. J Clin Endocrinol Metab. 2014 Oct;99(10):E1854-61.

13 Ueland T, Otterdal K, Lekva T, Halvorsen B, Gabrielsen A, Sandberg WJ, et al. Dickkopf-1 enhances inflammatory interaction between platelets and endothelial cells and shows increased expression in atherosclerosis. Arterioscler Thromb Vasc Biol. 2009 Aug;29(8):1228-34.

14 Mill C, George SJ. Wnt signalling in smooth muscle cells and its role in cardiovascular disorders. Cardiovasc Res. 2012 Jul;95(2):233-40.

15 Goliasch G, Wiesbauer F, Kastl SP, Katsaros KM, Blessberger H, Maurer G, et al. Premature myocardial infarction is associated with low serum levels of Wnt-1. Atherosclerosis. 2012 May;222(1):251-6.

16 Register TC, Hruska KA, Divers J, Bowden DW, Palmer ND, Carr JJ, et al. Plasma Dickkopf1 (DKK1) concentrations negatively associate with atherosclerotic calcified plaque in African-Americans with type 2 diabetes. J Clin Endocrinol Metab. 2013 Jan;98(1):E60-5.

17 Jin S, Zhu M, Yan J, Fang Y, Lu R, Zhang W, et al. Serum sclerostin level might be a potential biomarker for arterial stiffness in prevalent hemodialysis patients. Biomarkers Med. 2016 Jul;10(7):689-99.

18 Kundakci Gelir G, Sengul S, Nergizoglu G, Ertürk S, Duman N, Kutlay S. Is Sclerostin Level Associated with Cardiovascular Diseases in Hemodialysis Patients? Blood Purif. 2018;46(2):118-25.

19 Hampson G, Edwards S, Conroy S, Blake GM, Fogelman I, Frost ML. The relationship between inhibitors of the Wnt signalling pathway (Dickkopf-1 (DKK1) and sclerostin), bone mineral density, vascular calcification and arterial stiffness in post-menopausal women. Bone. 2013 Sep;56(1):42-7.

20 Gaudio A, Fiore V, Rapisarda R, Sidoti MH, Xourafa A, Catalano A, et al. Sclerostin is a possible candidate marker of arterial stiffness: results from a cohort study in Catania. Mol Med Rep. 2017 May;15(5):3420-4.

21 Laurent S, Cockcroft J, Van Bortel L, Boutouyrie P, Giannattasio C, Hayoz D, et al.; European Network for Noninvasive Investigation of Large Arteries. Expert consensus document on arterial stiffness: methodological issues and clinical applications. Eur Heart J. 2006 Nov;27(21):2588-605.

22 DeLoach SS, Townsend RR. Vascular stiffness: its measurement and significance for epidemiologic and outcome studies. Clin J Am Soc Nephrol. 2008 Jan;3(1):184-92.

23 Mancia G, Fagard R, Narkiewicz K, Redón J, Zanchetti A, Böhm M, et al.; Task Force Members. 2013 ESH/ESC Guidelines for the management of arterial hypertension: the Task Force for the management of arterial hypertension of the European Society of Hypertension (ESH) and of the European Society of Cardiology (ESC). J Hypertens. 2013 Jul;31(7):1281-357.

24 Moe SM, Chen NX. Mechanisms of vascular calcification in chronic kidney disease. J Am Soc Nephrol. 2008 Feb; 19(2):213-6.

25 Nowak A, Artunc F, Serra AL, Pollock E, Krayenbühl PA, Müller C, et al. Sclerostin quo vadis? - is this a useful longterm mortality parameter in prevalent hemodialysis patients? Kidney Blood Press Res. 2015;40(3):266-76.

26 Delanaye P, Krzesinski JM, Warling X, Moonen M, Smelten N, Médart L, et al. Clinical and biological determinants of sclerostin plasma concentration in hemodialysis patients. Nephron Clin Pract. 2014;128(1-2):127-34.

27 Desjardins L, Liabeuf S, Oliveira RB, Louvet L, Kamel S, Lemke HD, et al.; European Uremic Toxin (EUTox) Work Group. Uremic toxicity and sclerostin in chronic kidney disease patients. Nephrol Ther. 2014 Nov;10(6):463-70.

28 Morena M, Jaussent I, Dupuy AM, Bargnoux AS, Kuster N, Chenine L, et al. Osteoprotegerin and sclerostin in chronic kidney disease prior to dialysis: potential partners in vascular calcifications. Nephrol Dial Transplant. 2015 Aug; 30(8):1345-56.

29 Delanaye P, Paquot F, Bouquegneau A, Blocki F, Krzesinski JM, Evenepoel P, et al. Sclerostin and chronic kidney disease: the assay impacts what we (thought to) know. Nephrol Dial Transplant. 2018 Aug;33(8):1404-10.

30 Hsu BG, Liou HH, Lee CJ, Chen YC, Ho GJ, Lee MC. Serum Sclerostin as an Independent Marker of Peripheral Arterial Stiffness in Renal Transplantation Recipients: A Cross-Sectional Study. Medicine (Baltimore). 2016 Apr; 95(15):e3300.

31 Thambiah S, Roplekar R, Manghat P, Fogelman I, Fraser WD, Goldsmith D, et al. Circulating sclerostin and Dickkopf-1 (DKK1) in predialysis chronic kidney disease (CKD): relationship with bone density and arterial stiffness. Calcif Tissue Int. 2012 Jun;90(6):473-80.

32 Zhu D, Mackenzie NC, Millán JL, Farquharson C, MacRae VE. The appearance and modulation of osteocyte marker expression during calcification of vascular smooth muscle cells. PLoS One. 2011;6(5):e19595.

33 Morales-Santana S, García-Fontana B, García-Martín A, Rozas-Moreno P, García-Salcedo JA, Reyes-García R, et al. Atherosclerotic disease in type 2 diabetes is associated with an increase in sclerostin levels. Diabetes Care. 2013 Jun;36(6):1667-74. 